\title{
A APLICAÇÃO DA PONTUAÇÃO RÁPIDA DE AVALIAÇÃO DE FALHA DE ÓRGÃOS SEQUENCIAIS (QSOFA) COMO MARCADOR DIAGNÓSTICO NA SEPSE: REVISÃO INTEGRATIVA
}

\author{
APPLICATION OF THE QUICK SEPSIS RELATED ORGAN FAILURE \\ ASSESSMENT (QSOFA) AS A DIAGNOSTIC MARKEN IN SEPSIS: \\ INTEGRATIVE REVIEW
}

\author{
Karoliny Rodrigues Rosa ${ }^{1}$ \\ Macerlane de Lira Silva ${ }^{2}$ \\ Renata Livia Silva Fonsêca Moreira de Medeiros ${ }^{3}$ \\ Francisco Carlos de Oliveira Júnior ${ }^{4}$
}

RESUMO: OBJETIVO: Esse trabalho possui como objetivo analisar, a partir da literatura pertinente, a aplicação do qSOFA como um marcador de diagnóstico na sepse. METODOLOGIA: O levantamento bibliográfico foi realizado pela internet nas bases de dados: Biblioteca Nacional em Saúde (BVS), Biblioteca Digital da UNB (BDM), Scientific Electronic Library Online (SciELO), Medical Literature Analysis and Retrieval System On-line (MEDLINE) e National Library of Medicine (Pubmed), entre os anos de 2003 a 2018. Nove artigos foram selecionados de acordo com os parâmetros de inclusão e exclusão. RESULTADOS E DISCUSSÃO: Como critério de triagem à beira do leito, pacientes adultos com suspeita de infecção podem ser rapidamente identificados como mais propensos a ter maus resultados típicos de sepse se tiverem pelo menos dois pontos no novo escore rápido qSOFA. $O$ resultado QSOFA será positivo nos casos em que, no paciente, for evidenciada a FR> 22/incursões por minuto, nível de consciência inferior a 15 na escala de

\footnotetext{
${ }^{1}$ Acadêmica do Curso de Medicina da Faculdade de Santa Maria Cajazeiras - PB.

${ }^{2}$ Graduado em Enfermagem pela Faculdade Santa Maria Cajazeiras - PB (2007), especialização em política e gestão do cuidado com ênfase no apoio matricial pela UFPB e mestrado em Saúde Coletiva pela UNISANTOS. Docente da Faculdade Santa Maria de Cajazeiras, membro do comitê de Ética em Pesquisa da Universidade Federal de Campina Grande, campus Cajazeiras.

${ }^{3}$ Enfermeira pela Universidade Federal da Paraíba - UFBP, Docente da Faculdade Santa Maria FSM, Especialista em Saúde Pública pela FACISA, Mestre em Enfermagem pela Universidade Federal da Paraíba - UFPB, Doutora em Pesquisa em Cirurgia pela Faculdade de Ciências Médicas Santa Casa de São Paulo - FCMSP.

${ }^{4}$ Médico pela UNIVERSIDADE FEDERAL DE CAMPINA GRANDE (2008), com Especialização em Medicina Intensiva. Docente da disciplina Urgência e Emergência e Hematologia na Faculdade Santa Maria, PB.
} 
Glasgow ou quando a pressão arterial sistólica for <que $100 \mathrm{mmHg}$. No entanto, isto não é pacificado, pois, nos casos de suspeita de sepse, as características a serem consideradas baseiam-se na presença de algum dos critérios, como hipotensão, seja a pressão arterial sistólica $<90 \mathrm{mmHg}$ ou PAM $<65 \mathrm{mmHg}$ ou queda de PA > 40 mmHg; oligúria; elevação da creatinina; relação $\mathrm{PaO} 2 / \mathrm{FiO} 2<300$ ou necessidade de $\mathrm{O} 2$ para manter $\mathrm{SpO} 2>90 \%$; contagem de plaquetas < $100.000 / \mathrm{mm}^{3}$ ou redução de $50 \%$ no número de plaquetas em relação ao maior valor registrado nos últimos três dias; acidose metabólica: déficit de bases $\leq 5,0 \mathrm{mEq} / \mathrm{l}$. CONSIDERAÇÕES FINAIS: A sepse caracteriza-se por uma reação desordenada do organismo desencadeando em uma série de infecções. Conclui-se que são necessários rápida avaliação e diagnóstico sobre a sepse, ademais, o indicador SOFA mostrou-se mais completo do que o qSOFA. Os novos critérios mostraram-se indicadores necessários para o diagnostico da sepse, demonstrando, assim, a necessidade da rapidez de sua aplicação para que o paciente venha a obter êxito na sua recuperação, e esta doença tão grave pare de acometer um maior número de vítimas.

PALAVRAS CHAVE: Diagnóstico; Sepse; qSOFA.

ABSTRACT: OBJECTIVE: The objective of this work is to analyze, from relevant literature, the application of the qSOFA as a diagnostic marker in sepsis. METHODOLOGY: The bibliographic survey was performed through the internet at the databases: National Health Library (VHL), UNB Digital Library (BDM), Scientific Electronic Library Online (SciELO), Medical Literature Analysis and Retrieval System Online (MEDLINE) and National Library of Medicine (Pubmed), from 2003 to 2018. Nine articles were selected according to the inclusion and exclusion parameters. RESULTS AND DISCUSSION: As a bedside screening criterion, adult patients with suspected infection can be quickly identified as more likely to having typical poor sepsis outcomes if they have at least two points in the new qSOFA rapid score. The QSOFA result will be positive when the patient presents $R R>22 /$ raids per minute, consciousness level below 15 on the Glasgow scale, or when systolic blood pressure is $<100 \mathrm{mmHg}$. However, this is not pacified because, in cases of suspected sepsis, the characteristics to be considered are based on the presence of any of the criteria, such as hypotension, either systolic blood pressure $<90 \mathrm{mmHg}$ or MAP $<65 \mathrm{mmHg}$ or decreased $\mathrm{BP}>40 \mathrm{mmHg}$; oliguria; creatinine elevation; $\mathrm{PaO}_{2} / \mathrm{FiO}_{2}$ ratio $<300$ or $\mathrm{O} 2$ requirement to maintain $\mathrm{SpO}_{2}>90 \%$; platelet count $<100,000 / \mathrm{mm}^{3}$ or $50 \%$ reduction in platelet count over the highest value recorded in the last three days; metabolic acidosis: base deficit $\leq 5.0 \mathrm{mEq} / \mathrm{l}$. FINAL THOUGHTS: Sepsis is characterized by a disordered reaction of the body triggering a series of infections. The rapid assessment and diagnosis of sepsis are necessary, in addition, the SOFA indicator was more complete than qSOFA. The new criteria have proved to be necessary indicators for the diagnosis of sepsis, thus demonstrating the need for rapid application so that the patient will be able to successfully recover, and this serious disease ceases to affect a greater number of victims.

Keywords: Diagnosis; Sepsis; qSOFA. 


\section{INTRODUÇÃO}

A palavra sepse deriva-se etimologicamente do grego septikós, conceituado por Hipócrates (460-377 a.C), significando apodrecer, isto é, o que causa a putrefação. À época, este termo era correspondente a um quadro clínico em que "a superfície externa do corpo era fria, com febre contínua, internamente, e alta sensação de calor e sede". Na Grécia antiga, a sepse era diretamente relacionada à putrefação e estava associada a doenças e mortes. Posteriormente, a sepse foi compreendida como um evento benéfico, visto que era necessário para a cicatrização de feridas. Semmelweis, ao confirmar a sua teoria dos germes, como também Pasteur e demais pesquisadores, redefiniram a sepse como sendo uma infecção sistêmica, comumemente atribuida a um envenenamento do sangue, acreditando-se que organismos patogênicos em um organismo hospedeiro tinham se espalhado na corrente sanguínea. Entretanto, com o avanço da ciência, a criação de antibióticos modernos, a teoria dos germes não conseguiu explicar a patogênese da sepse, posto que muitos pacientes morreram, embora tenha ocorrido o êxito na erradicação do agente patogênico desencadeante (KEMPKER et al., 2018).

Então, a sepse foi compreendida como uma condição clínica que resulta de uma infecção bacteriana, e a septicemia, a existência destas bactérias na corrente sanguínea. A partir de então, diversas foram as terminologias da sepse para denominar a deterioração clínica dos pacientes em choque nas emergências e Unidades de Terapia Intensiva (UTIs) (MEHTA et al., 2017).

Em 1991, ao realizarem uma conferência, o Colégio Americano de Pneumologia e a Sociedade de Medicina Intensiva (ACCP/SCCM) desenvolveram as definições iniciais da sepse, compreendida como uma resposta inflamatória à infecção, fazendo com que haja disfunções no organismo, em decorrência da síndrome inflamatória sistêmica (SRIS) atribuída a um foco infeccioso. Desta feita, quatro critérios SRIS foram adotados, denominados como taquicardia, taquipneia, hipotermia e leucopenia (GUL et al., 2017). Para Finkelsztein et al. (2017), ao 
desenvolver estudos acerca do presente tema, estes critérios são insuficientes na identificação de pacientes infectados em risco de um curso complicado.

Hodiernamente, a sepse é compreendida como uma condição clínica ocasionada por uma resposta inflamatória desregulada, fazendo com que haja disfunções no organismo (TAEB; HOOPER; MARIK, 2017). Este desequilibrio ocorre em organismos endógenos pró e anti-inflamatórios, sendo responsável pela proliferação de fenômenos que desencadeiam disfunções orgânicas (SOGAYAR, 2008).

De acordo com um estudo epidemiológico da sepse realizado em diversas Unidades de Terapia Intensiva (UTI) do Brasil, foi comprovado que as principais áreas de infecção foram o trato respiratório e o abdômen (SALES JUNIOR et al., 2006).

Vale ressaltar que a sepse não se trata de uma doença específica, mas, sim, de uma síndrome que possui a sua patogenicidade na incerteza. Atualmente, pode ser identificada por uma infinidade de sinais e sintomas clínicos em um paciente com suspeita de infecção. Como não existe um teste de diagnóstico padrão ouro, vários grupos tentaram formular definições e fornecer critérios clínicos para a identificação de pacientes infectados em risco de mortalidade significativa (SINGER et al., 2016).

Em todo o globo, a sepse é considerada como uma importante causa de hospitalização, sendo a principal causa de mortes em UTIs (SANTOS et al., 2015), como também um grande desafio para os médicos (INSTITUTO LATINO AMERICANO DA SEPSE, 2016). Devido a ser uma das doenças que mais causam mortalidade no mundo, possuindo incidência de mais de 15 milhões de pessoas por ano, deve ser encarada como um dos principais problemas de saúde pública do globo (TORIO; ANDREWS, 2013). No Brasil, a sua mortalidade é frequente, posto que alcança quase $60 \%$, diferentemente da média mundial, que se encontra em torno de 30\% (SITNIK et al., 2014; SIQUEIRA-BATISTA et al., 2011).

Devido ao baixo número de marcadores biológicos ou clínicos para o diagnóstico da sepse, e diante da necessidade de adquirir as suas definições, as sociedades americanas e europeia de medicina intesiva, mobilizaram-se com o intuito de encontrar uma nova definição para a sepse, posto que os critérios desenvolvidos em 1991 não eram suficientes. Assim, a sepse é denominada como 
Sepsis-3, sendo conceituada como uma disfunção orgânica potencialmente fatal em decorrência de um hospedeiro desregulado à infecção, como também descarta a SRIS dos novos critérios (SEYMOUR et al., 2016).

Portanto, o critério atual de diagnóstico da sepse sugere que haja uma avaliação do escore de disfunção orgânica, a Sequential Organ Failure Assessment Score (SOFA), caracterizado em razão do aumento agudo de, no mínimo, dois pontos nesse escore (FINKELSZTEIN et al., 2017).

Vale salientar que o uso de pontuação do SOFA em ensaios clínicos já é comumente realizado, sendo um componente de rotina de coleta de dados para ensaios clínicos na unidade de terapia intensiva (UTI), conforme Marik e Taeb (2017). Entretanto, para a sua realização, faz-se necessária a realização de exames laboratóriais que nem sempre estarão disponíveis celeremente, e, assim, o seu uso só é comum dentro da unidade de terapia intensiva.

Desta forma, de acordo com as limitações existentes, no intuito de otimizar o atendimento dos pacientes pré-hospitalares bem como os de emergência, as sociedades de medicina sugeriram a criação de um escore denominado de Pontuação Rápida de Avaliação de Falhas de Órgãos Sequenciais (quick SOFA ou qSOFA) para avaliação da disfunção orgânica (FINKELSZTEIN et al., 2017).

Embora exista uma série de limitações à aplicabilidade do qSOFA, este novo escore é uma ferramenta mais simples, que atende determinadas variáveis negligenciadas, a exemplo da consciência alterada, hipotensão e taquipneia. Assim, podem ser verificadas à beira do leito e identificadas rapidamente em pacientes adultos, possibilitando desfechos clínicos favoráveis, caso apresentem infecção. Além disso, ele vai definir os pacientes com suspeita de infecção que são susceptíveis de ter uma estadia de UTI prolongada ou maior risco de morte (SINGER et al., 2016). 


\section{OBJETIVO}

O presente trabalho possui o objetivo de realizar uma revisão integrativa acerca da aplicação da pontuação rápida de avaliação de falha de órgãos sequenciais (qSOFA) como marcador diagnóstico na sepse.

\section{METODOLOGIA}

Para a realização do presente trabalho, foi eleito o método da revisão integrativa da literatura, concebido como um modo de pesquisa amplo, posto que permite a síntese de diversos estudos publicados sobre o tema de interesse. Desse modo, é possível gerar novos conhecimentos de acordo com os resultados apresentados por pesquisas anteriores. Assim sendo, a análise de pesquisas relevantes dá suporte para a tomada de decisão e a melhoria da prática clínica, possibilitando a síntese de determinado assunto. (BOTELHO; DE ALMEIDA CUNHA; MACEDO, 2011; DAL SASSO MENDES; CAMPOS PEREIRA SILVEIRA; GALVÃO, 2008).

As etapas de desenvolvimento da revisão integrativa da literatura ocorrerão em seis modalidades, a saber: 1- estabelecer a hipótese e questão norteadora; 2delimitar a amostra a ser estudada, através dos critérios de inclusão e exclusão; 3categorizar os estudos; 4- avaliação dos estudos incluídos na revisão; 5- interpretar os resultados e 6- apresentar a síntese da revisão. (DAL SASSO MENDES; CAMPOS PEREIRA SILVEIRA; GALVÃO, 2008).

Diante da temática em questão, surge um questionamento utilizado como base para nortear toda a pesquisa: O qSOFA como marcador diagnóstico é uma ferramenta adequada para identificar a sepse?

O levantamento bibliográfico foi realizado através das bases de dados virtuais da Biblioteca Nacional em Saúde (BVS), Scientific Electronic Library Online 
(SciELO), Medical Literature Analysis and Retrieval System On-line (MEDLINE), National Library of Medicine (Pubmed) e Biblioteca Digital da UNB (BDM). Foram utilizados os Descritores em Ciências da Saúde (DECS): Qsofa, sepse/sepsis, diagnóstico/diagnosis.

Para o refinamento adequado da pesquisa, foi definida uma amostra, obedecendo aos seguintes critérios de inclusão: artigos que disponibilizassem seu texto completo, artigos com versão online gratuita, estudos de revisão, assunto principal, produções nacionais e internacionais, que estivessem publicados nos idiomas português e inglês, entre os anos de 2003 a 2018. Foram excluídos estudos que se repetiam nas bases de dados e artigos que não respondiam o objetivo do trabalho. Portanto, nove artigos foram selecionados de acordo com os parâmetros de inclusão e exclusão.

Para realização da análise dos artigos, criaram-se dois quadros, os quais contemplaram os seguintes aspectos respectivamente: título da pesquisa, ano de publicação/periódico e base de dados; autores, objetivos, metodologia e principais resultados. Após a leitura dos artigos, foi desenvolvida uma análise que norteou o tema estudado.

\section{RESULTADOS}

Os resultados encontrados acerca da aplicação da pontuação rápida de avaliação de falha de órgãos sequenciais (qSOFA) como marcador diagnóstico na sepse podem ser encontrados no Quadro 2. Torna-se imprescindível evidenciar o fato de existirem diversos índices de marcador de diagnóstico, bem como poucas vezes em que ocorre a sua utilização. 
Quadro 1: Apresentação da síntese de artigos incluídos na revisão integrativa.

Título/Ano/Base de dados.

\begin{tabular}{|c|c|c|c|}
\hline $\mathbf{N}^{\circ}$ & TÍTULO & ANO & $\begin{array}{l}\text { BASE DE } \\
\text { DADOS }\end{array}$ \\
\hline 1 & Avanços no diagnóstico e tratamento da sepse & 2003 & SCIELO \\
\hline 2 & $\begin{array}{l}\text { Nosocomial infections and bacterial resistance in patients } \\
\text { from a Teaching Hospital Intensive Care Unit. }\end{array}$ & 2007 & SCIELO \\
\hline 3 & Sepse: diagnóstico e tratamento & 2010 & BVS \\
\hline 4 & Estadiamento da Injúria Renal Aguda na Sepse & 2014 & BDM \\
\hline 5 & $\begin{array}{l}\text { Implementation of a multifaceted sepsis education program } \\
\text { in an emerging country setting: clinical outcomes and cost- } \\
\text { effectiveness in a long-term follow-up study. }\end{array}$ & 2014 & SCIELO \\
\hline 6 & $\begin{array}{l}\text { The Third International Consensus Defi initions for Sepsis } \\
\text { and Septic Shock (Sepsis-3) }\end{array}$ & 2016 & PUBMED \\
\hline 7 & Dear Sepsis-3 we are sorry to say that we don't like you. & 2017 & SCIELO \\
\hline 8 & $\begin{array}{l}\text { Diagnóstico de sepse em pacientes após internação em } \\
\text { unidade de terapia intensiva }\end{array}$ & 2017 & SCIELO \\
\hline 9 & $\begin{array}{l}\text { Association of the Quick Sequential (Sepsis-Related) } \\
\text { Organ Failure Assessment (qSOFA) Score With Excess } \\
\text { Hospital Mortality in Adults With Suspected Infection in } \\
\text { Low- and Middle-Income Countries. }\end{array}$ & 2018 & SCIELO \\
\hline
\end{tabular}

Quadro 2: Apresentação da síntese de artigos incluídos na revisão integrativa. Autores/Objetivos/Metodologia/Resultados.

\begin{tabular}{|c|c|c|c|c|}
\hline $\mathbf{N}^{\circ}$ & AUTORES & OBJETIVOS & METODOLOGIA & RESULTADOS \\
\hline 1 & $\begin{array}{l}\text { CARVALHO, } \\
\text { Paulo R.A.; } \\
\text { TROTTA, } \\
\text { Eliana de A. }\end{array}$ & $\begin{array}{l}\text { Discutir os } \\
\text { avanços acerca } \\
\text { do diagnóstico e } \\
\text { tratamento da } \\
\text { sepse. }\end{array}$ & $\begin{array}{ll}\text { Revisão } & \\
\text { bibliográfica e } & \text { de } \\
\text { literatura sobre a } \\
\text { sepse e } & \text { os } \\
\text { aspectos } & \text { de } \\
\text { diagnosticos } & \\
\text { terapeuticos. }\end{array}$ & $\begin{array}{l}\text { Em conclusão, } \\
\text { diagnóstico da sepse é } \\
\text { tão desafiador, pois, } \\
\text { quando não identificado } \\
\text { rapidamente, pode } \\
\text { culminar até em morte. } \\
\text { Um dos fatores que } \\
\text { torna esta identificação } \\
\text { tão complicada é a } \\
\text { semelhança de sintomas } \\
\text { com demais doenças }\end{array}$ \\
\hline
\end{tabular}




\begin{tabular}{|c|c|c|c|c|}
\hline & & & & não infecciosas \\
\hline 2 & $\begin{array}{l}\text { OLIVEIRA, } \\
\text { Adriana } \\
\text { Cristina et al. }\end{array}$ & 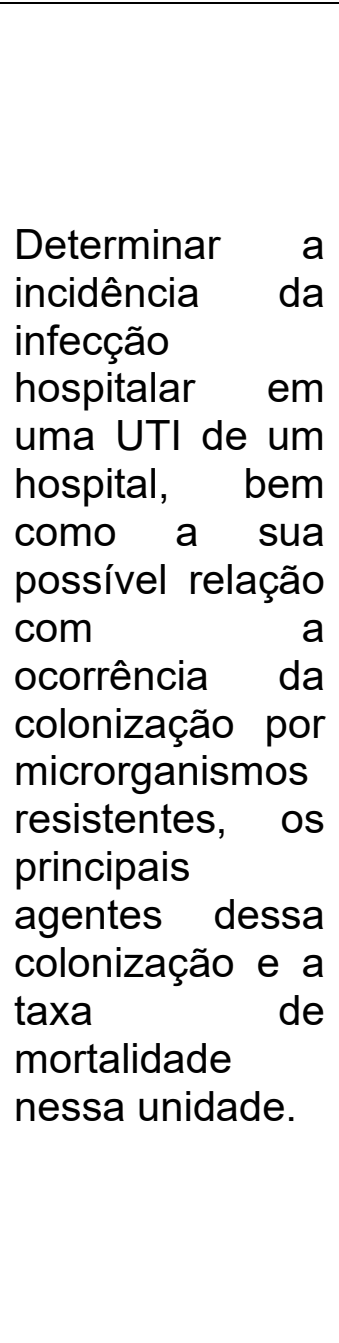 & $\begin{array}{l}\text { Pesquisa } \\
\text { quantitativa } \\
\text { através do estudo } \\
\text { de casos. }\end{array}$ & $\begin{array}{l}\text { O estudo prospectivo e } \\
\text { descritivo foi realizado } \\
\text { no período de agosto de } \\
2005 \text { a julho de } 2006 \text {. } \\
\text { Os dados foram } \\
\text { coletados dos } \\
\text { prontuários e consultas } \\
\text { ao banco de dados } \\
\text { microbiológicos. } \\
\text { Constatou-se a taxa } \\
\text { global de infecção de } \\
23,8 \% \text { a colonização } \\
\text { por microrganismos } \\
\text { resistentes contribuiu } \\
\text { para o desenvolvimento } \\
\text { da infecção (RR = } 6,8 \\
\text { [4,8-9,6], p <0,05), os } \\
\text { patógenos resistentes } \\
\text { mais prevalentes foram } \\
\text { Pseudomonas } \\
\text { aeruginosa } \\
\text { Acinetobacter } \\
\text { baumannii. A taxa de } \\
\text { mortalidade foi de 10,8\% } \\
\text { e destes, 23 pacientes } \\
\text { (37,1\%) morreram após } \\
\text { o diagnóstico de } \\
\text { infecção hospitalar }\end{array}$ \\
\hline 3 & $\begin{array}{l}\text { Marcelo } \\
\text { BOECHAT, } \\
\text { Antônio Luiz; } \\
\text { BOECHAT, } \\
\text { Narjara de } \\
\text { Oliveira. }\end{array}$ & $\begin{array}{l}\text { Contextualizar o } \\
\text { tratamento da } \\
\text { sepse, bem } \\
\text { como revisar os } \\
\text { principais } \\
\text { aspectos da } \\
\text { epidemiologia } \\
\text { da sepse no } \\
\text { Brasil e os } \\
\text { tratamentos com } \\
\text { base na } \\
\text { Campanha } \\
\text { Sobrevivendo a } \\
\text { Sepse. }\end{array}$ & $\begin{array}{l}\text { Revisão } \\
\text { bibliográfica } \\
\text { integrativa. }\end{array}$ & $\begin{array}{l}\text { Surgiu, em 2003, a } \\
\text { Campanha } \\
\text { Sobrevivendo a Sepse, } \\
\text { sugerindo } \\
\text { implementação } \\
\text { critérios de } \\
\text { tratamento e detecção } \\
\text { precoce da sepse, com } \\
\text { o objetivo de reduzir sua } \\
\text { mortalidade bem como } \\
\text { melhorar o prognóstico } \\
\text { dos pacientes já } \\
\text { acometidos por essa } \\
\text { patologia, dando ênfase } \\
\text { nos pacotes de } \\
\text { tratamento. }\end{array}$ \\
\hline 4 & $\begin{array}{l}\text { GOMES, } \\
\text { Thaís } \\
\text { Martins } \\
\end{array}$ & $\begin{array}{lr}\text { Identificar } & a \\
\text { incidência } & \text { de } \\
\text { pacientes } & \text { com } \\
\end{array}$ & $\begin{array}{l}\text { Pesquisa } \\
\text { quatitativa com } \\
\text { coleta de dados e }\end{array}$ & $\begin{array}{llr}\text { A } & \text { atuação } & \text { do } \\
\text { profissional de } & \text { saúde } \\
\text { poderá ser de } & \text { forma }\end{array}$ \\
\hline
\end{tabular}




\begin{tabular}{|c|c|c|c|c|}
\hline & & $\begin{array}{lr}\text { sepse } & \text { que } \\
\text { evoluem } & \text { com } \\
\text { injúria } & \text { renal } \\
\text { aguda } & \text { no } \\
\text { cenário } & \text { de } \\
\text { terapia } & \\
\text { intensiva. } & \end{array}$ & $\begin{array}{l}\text { abordagem } \\
\text { descritiva. }\end{array}$ & $\begin{array}{l}\text { diferencial, através do } \\
\text { planejamento de ações } \\
\text { ao utilizar os } \\
\text { instrumentos a exemplo } \\
\text { da NIC (classificação } \\
\text { das intenvernções em } \\
\text { enfermagem) para que } \\
\text { seja possível prevenir } \\
\text { choque, controlar a } \\
\text { infecção, controlar o } \\
\text { ácido-base, controlar a } \\
\text { volemia, ter cuidados } \\
\text { específicos com o } \\
\text { coração, como também } \\
\text { a prevenção de } \\
\text { embolias e } \\
\text { monitoramento } \\
\text { respiratório. }\end{array}$ \\
\hline 5 & $\begin{array}{l}\text { NORITOMI, } \\
\text { Danilo } \\
\text { Teixeira et } \\
\text { al. }\end{array}$ & $\begin{array}{lr}\text { Realizar } & \text { um } \\
\text { estudo } & \\
\text { prospectivo } & \text { e } \\
\text { multicêntrico } & \\
\text { acerca } & \text { da } \\
\text { letalidade da } & \text { depse no Brasil. }\end{array}$ & $\begin{array}{l}\text { Pesquisa } \\
\text { quantitativa com } \\
\text { coleta de dados, } \\
\text { e abordagem } \\
\text { descritiva. }\end{array}$ & $\begin{array}{l}\text { Verificou-se que a } \\
\text { letalidade da doença no } \\
\text { Brasil é de } 67,4 \% \text {, } \\
\text { número alarmante } \\
\text { quando comparado com } \\
\text { demais países do globo, } \\
\text { a exemplo da Alemanha } \\
\text { (43,4\%), Índia (39\%) e } \\
\text { Austrália }(43,4 \%) .\end{array}$ \\
\hline 6 & $\begin{array}{l}\text { SINGER, } \\
\text { Mervyn et al. }\end{array}$ & $\begin{array}{l}\text { Conceituar a } \\
\text { sepse, bem } \\
\text { como } \\
\text { determinar o } \\
\text { uso do qSOFA } \\
\text { como indicador } \\
\text { para identificar a } \\
\text { Sepsis 3. }\end{array}$ & $\begin{array}{l}\text { Revisão } \\
\text { integrativa } \\
\text { realizada por } \\
\text { meio de uma } \\
\text { pesquisa } \\
\text { bibliográfica. }\end{array}$ & $\begin{array}{l}\text { A sepsis } 3 \text { é a "presença } \\
\text { de disfunção orgânica } \\
\text { ameaçadora à vida } \\
\text { secundária à resposta } \\
\text { desregulada } \\
\text { organismo à infecção". } \\
\text { Para o diagnóstico } \\
\text { clínico, a disfunção } \\
\text { orgânica pode ser } \\
\text { representada por um } \\
\text { aumento de dois pontos } \\
\text { ou mais pontos no } \\
\text { escore sofA. Como } \\
\text { critério de triagem à } \\
\text { beira do leito, pacientes } \\
\text { adultos com suspeita de } \\
\text { infecção podem ser } \\
\text { rapidamente } \\
\text { identificados como mais } \\
\text { propensos a ter maus } \\
\text { resultados típicos de }\end{array}$ \\
\hline
\end{tabular}




\begin{tabular}{|c|c|c|c|c|}
\hline & & & & $\begin{array}{l}\text { sepse se tiverem pelo } \\
\text { menos dois pontos no } \\
\text { novo escore rápido } \\
\text { quickSOFA ou qSOFA. } \\
\text { Esses critérios clínicos } \\
\text { atualizados } \\
\text { facilitar } \\
\text { reconhecimento precoce } \\
\text { e o gerenciamento mais } \\
\text { oportuno de pacientes } \\
\text { com sepse ou com risco } \\
\text { de desenvolver sepse. }\end{array}$ \\
\hline 7 & $\begin{array}{l}\text { CARNEIRO, } \\
\text { Antonio } \\
\text { Henriques; } \\
\text { PÓVOA, } \\
\text { Pedro; } \\
\text { GOMES, } \\
\text { José } \\
\text { Andrade. }\end{array}$ & $\begin{array}{l}\text { Apresentar os } \\
\text { novos conceitos } \\
\text { da sepse, } \\
\text { correlacionando- } \\
\text { a com a } \\
\text { síndrome da } \\
\text { resposta } \\
\text { inflamatória } \\
\text { sistêmica (SIRS) } \\
\text { bem como os } \\
\text { seus novos } \\
\text { critérios. }\end{array}$ & $\begin{array}{l}\text { Revisão } \\
\text { integrativa } \\
\text { realizada } \\
\text { meio de uma } \\
\text { pesquisa } \\
\text { bibliográfica. }\end{array}$ & $\begin{array}{l}\text { A compreensão dos } \\
\text { novos critérios utilizados } \\
\text { para a identificação da } \\
\text { sepse, somado aos } \\
\text { novos instrumentos para } \\
\text { melhor detecção } \\
\text { precoce e um raciocínio } \\
\text { clínico pautado no } \\
\text { embasamento científico. }\end{array}$ \\
\hline 8 & $\begin{array}{l}\text { MOURA, } \\
\text { Joyce } \\
\text { Marques et } \\
\text { al. }\end{array}$ & $\begin{array}{l}\text { Compreender as } \\
\text { características } \\
\text { clínicas como } \\
\text { também o } \\
\text { resultado dos } \\
\text { pacientes que } \\
\text { desenvolveram } \\
\text { sepse durante o } \\
\text { período de } \\
\text { internação em } \\
\text { uma unidade de } \\
\text { terapia } \\
\text { intensiva. }\end{array}$ & $\begin{array}{l}\text { Pesquisa } \\
\text { descritiva } \\
\text { abordagem } \\
\text { quantitativa. }\end{array}$ & $\begin{array}{l}\text { A padronização da } \\
\text { caracterização de } \\
\text { pacientes de UTI poderá } \\
\text { auxiliar nas diretrizes } \\
\text { das admissões e altas } \\
\text { da unidade, posto que o } \\
\text { conhecimento acerca do } \\
\text { perfil dos doentes } \\
\text { críticos beneficia o } \\
\text { estabelecimento de } \\
\text { critérios objetivos para } \\
\text { determinada finalidade. }\end{array}$ \\
\hline 9 & $\begin{array}{l}\text { RUDD KE, } \\
\text { SEYMOUR } \\
\text { CW, } \\
\text { ALUISIO AR } \\
\text { et al. }\end{array}$ & $\begin{array}{l}\text { Estudo acerca } \\
\text { da aplicação do } \\
\text { qSOFA em } \\
\text { diversos } \\
\text { ambientes, } \\
\text { como também } \\
\text { em um estudo } \\
\text { de coorte. }\end{array}$ & $\begin{array}{l}\text { Pesquisa } \\
\text { abordagem } \\
\text { quantitativa. }\end{array}$ & $\begin{array}{l}\text { Foram analisados } 6218 \\
\text { pacientes hospitalizados } \\
\text { derivados de oito } \\
\text { estudos de coorte e um } \\
\text { estudo randomizado em } \\
\text { LMICs. Nessa análise, } \\
\text { os escores mais } \\
\text { elevados do qSOFA } \\
\text { foram associados a uma } \\
\text { maior mortalidade, mas } \\
\text { a validade preditiva }\end{array}$ \\
\hline
\end{tabular}




\begin{tabular}{|l|l|l|}
\hline & $\begin{array}{l}\text { variou significativamente } \\
\text { entre as coortes, } \\
\text { limitando a interpretação } \\
\text { dos resultados. É } \\
\text { importante ressaltar que } \\
\text { o escore de QSOFA é } \\
\text { um escore de disfunção } \\
\text { orgânica. Náo } \\
\text { diagnóstico de sepse } \\
\text { nem identifica aqueles } \\
\text { cuja disfunção orgânica } \\
\text { é devido À infecção, } \\
\text { mas ajuda a identificar } \\
\text { pacientes que } \\
\text { potencialmente têm um } \\
\text { alto risco de morrer de } \\
\text { infecção. }\end{array}$ \\
\hline
\end{tabular}

\section{DISCUSSÃO}

Em fevereiro do ano de 2006, a Society of Critical Care Medicine (SCCM) juntamente com a European Society of Critical Care Medicine (ESICM) conceituaram a nova definição de sepse, levando em consideração que anteriormente era conhecida como Síndrome de Inflamação Respiratória Sistêmica (SIRS), e hoje, como a avaliação da disfunção orgânica, analisando o aumento de no mínimo dois itens no escore Sequential Organ Failure Assessment (SOFA). Isto quer dizer que pacientes somente com escala de coma de Glasgow abaixo de 13 ou com hipotensão não se enquadrariam como sepse (CARNEIRO; PÓVOA; GOMES, 2017).

Assim sendo, deverá ser considerado o resultado QSOFA positivo nos casos em que, no paciente, for evidenciada a FR> 22/incursões por minuto, nível de consciência inferior a 15 na escala de Glasgow ou quando a pressão arterial sistólica for <que $100 \mathrm{mmHg}$. Desta feita, a sepse deverá ser conceituada como grave, entretanto, a "sepse grave" é uma definição excluída (MOURA et al., 2017).

No entendimento de Machado et al. (2016), o choque séptico adotado pela nova nomenclatura é definido nos casos em que o paciente não só apresenta a 
sepse, mas também há evidências de irregularidades circulatórias agudas e metabólicas juntamente com elevado risco de óbito. Os aspectos para a percepção do choque séptico ocorrem diante da necessidade de administrar vasopressor, visando a estabilizar a pressão arterial média acima de $65 \mathrm{mmHg}$, juntamente com nível sérico de lactato acima de $2 \mathrm{mmol} / \mathrm{l}$ posterior à reanimação volêmica adequada, porém, quando indicada a existência de hiperlactatemia isolada, sem considerar os níveis, não há critério de disfunção de acordo com o novo consenso.

No entanto, em 2016, o Instituto Americano Latino da Sepse (ILAS) opôs-se às novas definições, posto que as alterações não abrangem de forma adequada a real necessidade de diagnóstico em países subdesenvolvidos, como é o caso do Brasil. Porém, a Campanha de Sobrevivência de Implementação da Sepse (CSS) determinou que não irá modificar os requisitos utilizados na caracterização da disfunção orgânica no programa de qualidade, mantendo inclusive a hiperlactatemia como um dos critérios de avaliação (INSTITUTO AMERICANO LATINO DA SEPSE, 2016).

Nos casos de suspeita de sepse, os critérios a serem considerados baseiamse na presença de algum dos critérios, como hipotensão, seja a pressão arterial sistólica < $90 \mathrm{mmHg}$ ou PAM < $65 \mathrm{mmHg}$ ou queda de PA > $40 \mathrm{mmHg}$; oligúria; elevação da creatinina; relação $\mathrm{PaO} 2 / F i O 2<300$ ou necessidade de $\mathrm{O}_{2}$ para manter $\mathrm{SpO}_{2}>90 \%$; contagem de plaquetas $<100.000 / \mathrm{mm}^{3}$ ou redução de $50 \%$ no número de plaquetas em relação ao maior valor registrado nos últimos três dias; acidose metabólica: déficit de bases $\leq 5,0 \mathrm{mEq} / \mathrm{l}$ e lactato acima do valor de referência; rebaixamento do nível de consciência, aumento significativo de bilirrubinas (MACHADO, et al. 2016).

Para Gomes (2014), os profissionais de saúde devem atentar-se às distintas formas que a sepse pode se apresentar, evoluindo posteriormente para um quadro de elevado potencial de mortalidade, os sinais surgem como: choque séptico, diminuição da saturação do oxigênio, confusão, agitação, oligúria e hipotensão. Desse modo, quando o prognóstico for grave, pode culminar na falência múltipla de órgãos, hemorragias, coma e choque séptico, levando o paciente ao óbito.

É sabido que o bom prognóstico da sepse está diretamente ligado à detecção precoce, bem como à evolução clínica do paciente. Entretanto, ao tratar inicialmente 
o paciente, o profissional de saúde deve estar preparado para eventuais emergências que podem surgir na terapia intensiva. Com isso, considera-se que o protocolo da Campanha Sobrevivendo a Sepse coordenada pelo Instituto Latino Americano de Sepse e apoiada por diversas instituições de saúde, o qual está fundamentado na adesão aos protocolos de tratamento de três e seis horas (BOECHAT, 2010).

A campanha Sobrevivendo a Sepse estabeleceu como, primeiro tratamento, nas três primeiras horas do paciente acometido, a coleta de lactato sérico para avaliação do estado perfusional do paciente, a coleta de hemocultura antes do início da terapia com antibióticos, ventilação, início de antibioticoterapia, sendo este de largo espectro, e administrado por via endovenosa, reposição volêmica precoce em pacientes com hipotensão ou lactato acima de duas vezes o valor de referência (INSTITUTO LATINO AMERICANO DA SEPSE, 2016).

Boechat (2010) afirma que a estratégia de ventilação no paciente séptico coaduna com a utilização de volume corrente (VC) em concentrações baixas, a exemplo, em média de $6 \mathrm{ml} / \mathrm{kg}$ de peso ideal, e para aqueles que, além da sepse, progridem com lesão pulmonar aguda ou síndrome da angústia respiratória aguda (SARA), o indicado é pressão de platô até $30 \mathrm{cmH}_{2} \mathrm{O}$. Nos casos de intervenção com a pressão expiratória final positiva entre 8 e $15 \mathrm{cmH}_{2} \mathrm{O}$, é preferivel para os pacientes com hipoxemia refratária e que apresentam elevadas frações inspiradas de oxigênio.

Com o intuito de uniformizar a pontuação de avaliação para pacientes fora da UTI, buscando facilitar a identificação de pacientes em potencial risco de morrer de sepse, a comunidade médica norte americana, juntamente com a europeia, uniformizou a referida pontuação, conceituando que a identificação de sepse precoce é feita através da versão modificada do escore de SOFA, denominado escore rápido de avaliação de falha de órgãos sequencias quick-SOFA (qSOFA). Este escore é composto de apenas três componentes, sendo eles: Pressão arterial sistólica $(P A S) \leq 100 \mathrm{mmHg}$, frequência respiratória $(F R) \geq 22$ irpm e alteração do nível de consciência, ressaltando a facilidade em que cada componente pode ser verificado à beira do leito (SINGER et al., 2016). 
Devido ao qSOFA necessitar apenas de um exame clínico, a sua aplicação pode ocorrer em diversos ambientes, como enfermaria, emergência e UTI. O valor do qSOFA em países em desenvolvimento foi objeto de análise de 6218 pacientes internados derivados de oito estudos de coorte e um estudo randomizado em lower middle income countries (LMICs). Na referida análise, os escores mais elevados do qSOFA foram associados a uma maior mortalidade, porém a validade preditiva obteve variação significativa entre as coortes, cabendo a interpretação dos resultados. Cumpre salientar que o escore QSOFA é um escore de disfunção orgânica, e, desse modo, não é diagnóstico de sepse nem identifica aqueles cuja disfunção orgânica é devido à infecção, mas ajuda a identificar pacientes que potencialmente têm um alto risco de morrer de infecção. Ademais, não estabelece estratégias isoladas de tratamento nem pressente a mortalidade baseando-se em dados demográficos como idade ou doenças crônicas (RUDD et al., 2018).

Conforme um estudo de prevalência realizado pelo Instituto Latino Americano de Sepse (ILAS) em cerca de 230 UTIs brasileiras, comprovou-se que $30 \%$ dos leitos ocupados são por pacientes com choque séptico ou sepse.

Diante disto, a rapidez na identificação bem como no diagnóstico da disfunção orgânica e seu tratamento estão diretamente relacionados com o prognóstico do paciente (INSTITUTO LATINO AMERICANO DA SEPSE, 2016).

No mesmo sentido, um estudo prospectivo e multicêntrico verificou que a letalidade da doença no Brasil foi de $67,4 \%$, sendo discrepante da porcentagem de outros países, como Alemanha (43,4\%), Austrália (32,6\%) e Índia (39\%) (NORITOMI et al., 2014).

O diagnóstico da sepse precisa superar muitos desafios, posto que, quando não identificada precocemente, poderá acarretar em choque, falência de órgãos e até mesmo em morte. Vale ressaltar que um dos motivos pelos quais o diagnóstico da sepse não é simples está relacionado ao fato de que as primeiras manifestações clínicas são facilmente confundidas com sintomas de outros processos não infecciosos, bem como os indicadores laboratoriais indiretos, comumente utilizados para confirmar suspeita da sepse, sozinhos, possuem baixa especificidade (CARVALHO; TROTTA, 2003). 
Ademais, os critérios, bem como as definições para choque séptico e sepse, sofreram diversas revisões e alterações, específicamente em 1991, 2001 e 2016. Esta última atualização, ao pubicar a 'sepsis 3', abordou pontos positivos e negativos. Entre os destaques positivos, é possível citar a extinção do termo "sepse grave", posto que toda sepse é grave e lesiva. Dentre os pontos negativos, é possível evidenciar o aumento da especificidade e a redução da sensibilidade dos critérios, objeto de diversas críticas por parte dos autores, que consideram inapropriados diante da realidade dos países que possuem recursos limitados (MACHADO et al., 2016).

Cristalino observou que vários esforços foram feitos nos últimos anos com o intuito de melhorar a identificação da sepse. Ao analisar o quadro desta doença no Brasil, é de suma importância a avaliação da prevalência e dos fatores de risco da sepse, assim como da devida utilização de seus critérios diagnósticos (OLIVEIRA et al., 2007).

\section{CONSIDERAÇÕES FINAIS}

A sepse é definida como uma disfunção orgânica potencialmente fatal em decorrência de uma desregulada resposta inflamatória a uma infecção. Esta condição possui elevada importância visto que é responsável por um alto índice de mortalidade entre os pacientes internados em Unidades de Terapia Intensiva.

Desta feita, devido a sua relevância, a comunidade médica reuniu-se para estabelecer indicadores diante da ocorrência da sepse, posto que os seus sintomas podem ser facilmente confundidos com os de demais doenças não infecciosas.

Para a operacionalização clínica, a disfunção orgânica pode ser representada por um aumento no escore de avaliação de falha de órgãos sequencias (SOFA) de dois ou mais pontos. Entretanto, o SOFA adquiriu diversos entendimentos ao passar do tempo, motivo este que fez com que a padronização deste índice não fosse possível de realizar tão facilmente. 
Assim sendo, vale salientar que os principais critérios para avaliar disfunções orgânicas da sepse são: hipotensão (PAS < 90 mmHg ou PAM < 65 mmHg ou queda de $\mathrm{PA}>40 \mathrm{mmHg})$; oligúria $(\leq 0,5 \mathrm{ml} / \mathrm{Kg} / \mathrm{h})$ ou elevação da creatinina (>2mg/dl); relação $\mathrm{PaO}_{2} / \mathrm{FiO}_{2}<300$ ou necessidade de $\mathrm{O}_{2}$ para manter $\mathrm{SpO}_{2}>90 \%$; contagem de plaquetas $<100.000 / \mathrm{mm}^{3}$ ou redução de $50 \%$ no número de plaquetas em relação ao maior valor registrado nos últimos tres dias; lactato acima do valor de referência; rebaixamento do nível de consciência, agitação, delirium; aumento significativo de bilirrubinas ( $>2 \mathrm{X}$ o valor de referência).

Como uma forma de melhorar a triagem de pacientes sépticos em locais que não dispõem de recursos para realizar exames laboratoriais rapidamente e assim otimizar seu tratamento, foi criado um escore de triagem denominada rápida avaliação de falha de órgãos sequencias ("quick SOFA" ou qSOFA). Trata-se de uma ferramenta a ser utilizada à beira do leito para identificar rapidamente pacientes adultos, com suspeita de infecção, com maior probabilidade de terem desfechos clínicos desfavoráveis.

Desta feita, conclui-se que é necessaria a rápida avaliação e diagnóstico sobre a sepse, ademais, o indicador SOFA mostrou-se mais completo do que o qSOFA, este que serve para ser utilizado em países em desenvolvimento, devido as suas caracteristicas.

Assim, o objetivo do presente trabalho foi evidenciado, visto que o SOFA e o qSOFA mostraram-se indicadores necessários para o diagnóstico da sepse, demonstrando, assim, a necessidade da rapidez de sua aplicação para que o paciente venha a obter êxito na sua recuperação e esta doença tão grave pare de acometer um maior número de vítimas. 


\section{REFERÊNCIAS BIBLIOGRÁFICAS}

BOECHAT, A. L.; BOECHAT, N. O. Sepse: diagnóstico e tratamento. Revista brasileira clínica médica. São Paulo. v. 8, n. 5, p. 420-7, 2010. Disponível em: <http://files.bvs.br/upload/S/16791010/2010/v8n5/010.pdf>. Acesso em: 20 Out. 2019.

BOTELHO, L. L. R.; DE ALMEIDA CUNHA, C. C.; MACEDO, M. O método da revisão integrativa nos estudos organizacionais. Gestão e sociedade. v. 5, n. 11, p. 121-136, 2011.

CARNEIRO, A. H.; PÓVOA, P.; GOMES, J. A. Dear Sepsis-3, we are sorry to say that we don't like you. Revista brasileira de Terapia Intensiva. v. 29, n. 1, p. 4-8, 2017. Disponível em: $<$ http://www.scielo.br/scielo.php?script=sci_arttext\&pid=S0103-507X2017000100004>. Acesso em: 21 out. 2019.

CARVALHO, Paulo R.A.; TROTTA, Eliana de A. Avanços no diagnóstico e tratamento da sepse. J. Pediatr. v. 79, supl. 2, p. S195-S204, 2003.

MENDES, Karina Dal Sasso; SILVEIRA, Renata Cristina de Campos Pereira; GALVÃO, Cristina Maria. Revisão integrativa: método de pesquisa para a incorporação de evidências na saúde e na enfermagem. Texto \& contexto enfermagem. v. 17, n. 4, 2008.

FINKELSZTEIN, E. J. et al. Comparison of qSOFA and SIRS for predicting adverse outcomes of patients with suspicion of sepsis outside the intensive care unit. Critical Care. v. 21, n. 1, p. 73 , 2017. Disponivel em: <https://www.ncbi.nlm.nih.gov/pmc/articles/PMC5366240/>. Acesso em: 20 out. 2019.

GOMES, T. M. Estadiamento da Injúria Renal Aguda na Sepse. 2014. 62 f. Trabalho de Conclusão de Curso (Graduação) - Universidade de Brasília. Brasília, 2014. Disponível em: <http://bdm.unb.br/bitstream/10483/9912/1/2014_ThaisMartinsGomes.pdf>. Acesso em: 25 out. 2019.

GUL, F. Changing Definitions of Sepsis. Turk J Anaesthesiol Reanim. v. 45, p. 129- 38, 2017.

INSTITUTO LATINO AMERICANO DA SEPSE. Sepse: um problema de saúde pública. Disponível em:https://ilas.org.br/assets/arquivos/ferramentas/livro-sepse-um-problema-desaude-publica-cfm-ilas.pdf. Acesso em: 20 Out 2019.

KEMPKER, J. A.; WANG, H.; MARTIN, S. Sepsis is a preventable public health problem. Critical Care. p. 1-2, 2018.

MACHADO, Flavia Ribeiro et al. Getting a consensus: advantages and disadvantages of Sepsis 3 in the context of middle-income settings. Revista Brasileira de Terapia Intensiva. v. 28, n. 4 , p. $361-365,2016$.

MEHTA, Y.; KOCHHAR, G. Sepsis and Septic Shock. Medanta Institute of Critical Care and Anesthesiology. n. 1, p. 2-4, 2017.

MINASYAN, H. Sepsis and septic shock: Pathogenesis and treatment perspectives. Journal of Critical Care. v. 40, 2017.

MOURA, J. M. et al. Diagnóstico de sepse em pacientes após internação em unidade de terapia intensiva. Revista Arquivos Ciência Saúde. v. 24, n.3, p.55- 60, 2017. Disponível em: $<$ http://www.cienciasdasaude.famerp.br/index.php/racs/article/view/675>. Acesso em: 22 out 2019. 
NORITOMI, Danilo Teixeira et al. Implementation of a multifaceted sepsis education program in an emerging country setting: clinical outcomes and cost-effectiveness in a long-term follow-up study. Intensive care medicine. v. 40, n. 2, p. 182-191, 2014.

OLIVEIRA, Adriana Cristina et al. Nosocomial infections and bacterial resistance in patients from a Teaching Hospital Intensive Care Unit. Online Brazilian Journal of Nursing. v. 6, n. 2, ago. 2007. Disponível em: <http://www.objnursing.uff.br/index.php/nursing/article/view/837>. Acesso em: 22 out. 2019.

RUDD, K. E. et al. Association of the Quick Sequential (Sepsis-Related) Organ Failure Assessment (qSOFA) Score With Excess Hospital Mortality in Adults With Suspected Infection in Low- and Middle-Income Countries. JAMA. v. 319, n. 21, p. 2202-2211, 2018.

SALES JÚNIOR, J. A. L. et al. Sepse Brasil: estudo epidemiológico da sepse em unidades de terapia intensiva brasileiras. Rev Bras Ter Intensiva. v. 18, n. 1, p. 9- 17, 2006.

SANTOS, Alice Veras et al. Perfil epidemiológico da sepse em um hospital de urgência. Revista Prevenção de Infecção e Saúde. v. 1, n. 1, p. 19-30, 2015.

SEYMOUR, C. W. et al. Assessment of Clinical Criteria for Sepsis: For the Third International Consensus Definitions for Sepsis and Septic Shock (Sepsis-3). JAMA. v. 315, n. 8, p. 762-74, 2016. Disponível em: <https://www.ncbi.nlm.nih.gov/pubmed/26903335>. Acesso em: 22 out. 2019.

SINGER, Mervyn et al. The third international consensus definitions for sepsis and septic shock (sepsis-3). JAMA. v. 315, n. 8, p. 801-810, 2016.

SIQUEIRA-BATISTA, Rodrigo et al. Sepsis: an update. Revista Brasileira de terapia intensiva. v. 23, n. 2, p. 207-216, 2011.

SITNIK, Roberta et al. SeptiFast for diagnosis of sepsis in severely ill patients from a Brazilian hospital. Einstein. v. 12, n. 2, p. 191-196, 2014.

SOGAYAR, A. M. C. et al. A multicentre, prospective study to evaluate costs of septic patients in Brazilian intensive care units. Pharmacoeconomics. v. 26, n. 5, p. 425-434, 2008.

TAEB, Abdalsamih M.; HOOPER, Michael H.; MARIK, Paul E. Sepsis: Current Definition, Pathophysiology, Diagnosis, and Management. Nutrition in Clinical Practice. v. 32, n. 3, p. 296-308, $2017 . \quad$ Disponível em: <http://journals.sagepub.com/doi/abs/10.1177/0884533617695243>. Acesso em: 20 out. 2019.

TORIO, C. M.; ANDREWS, R. M. National inpatient hospital costs: the most expensive conditions by payer, 2011. Healthcare Cost and Utilization Project Statistical. 2013. Disponível em: https://www.hcup-us.ahrq.gov/reports/statbriefs/sb160.jsp . Acesso em: 20 Out 2019. 